\title{
Localization and Schrödinger Perturbations of Kernels
}

\author{
Krzysztof Bogdan • Wolfhard Hansen • \\ Tomasz Jakubowski
}

Received: 26 January 2012 / Accepted: 15 October 2012 / Published online: 17 November 2012

(C) The Author(s) 2012. This article is published with open access at Springerlink.com

\begin{abstract}
We study iterations of integral kernels satisfying a transience-type condition and we prove exponential estimates analogous to Gronwall's inequality. As a consequence we obtain estimates of Schrödinger perturbations of integral kernels, including Markovian semigroups.
\end{abstract}

Keywords Kernel-Absorbing set

Mathematics Subject Classifications (2010) 47 A55 • 60J35

\section{Introduction}

To motivate our results we consider the Gaussian transition density on $\mathbb{R}^{d}$,

$$
p(s, x, t, y)= \begin{cases}{[4 \pi(t-s)]^{-d / 2} \exp \frac{-|x-y|^{2}}{4(t-s)},} & \text { if } s<t, \\ 0, & \text { if } s \geq t\end{cases}
$$

The research was partially supported by grants MNiSW N N201 397137, MNiSW N N201 422539, ANR-09-BLAN-0084-01.

\footnotetext{
K. Bogdan

Institute of Mathematics of the Polish Academy of Sciences,

ul. Śniadeckich 8, 00-956 Warszawa, Poland

e-mail: bogdan@pwr.wroc.pl

W. Hansen

Fakultät für Mathematik, Universität Bielefeld, Postfach 100131, 33501 Bielefeld, Germany e-mail: hansen@math.uni-bielefeld.de

T. Jakubowski $(\bowtie)$

Institute of Mathematics and Computer Science, Wrocław University of Technology,

Wybrzeże Wyspiańskiego 27, 50-370 Wrocław, Poland

e-mail: Tomasz.Jakubowski@pwr.wroc.pl
} 
where $d \geq 1, s, t \in \mathbb{R}$ and $x, y \in \mathbb{R}^{d}$. Note that $-p$ is a left inverse of $\partial_{t}+\Delta_{y}$ :

$$
\int_{\mathbb{R}} \int_{\mathbb{R}^{d}} p(s, x, t, y)\left[\partial_{t} \phi(t, y)+\Delta_{y} \phi(t, y)\right] d y d t=-\phi(s, x), \quad \phi \in C_{c}^{\infty}\left(\mathbb{R} \times \mathbb{R}^{d}\right) .
$$

Let $q(t, y) \geq 0$ be a Borel function on $\mathbb{R} \times \mathbb{R}^{d}$. Let $p_{0}=p$, and for $n=1,2, \ldots$,

$$
p_{n}(s, x, t, y)=\int_{\mathbb{R}} \int_{\mathbb{R}^{d}} p_{n-1}(s, x, u, z) q(u, z) p(u, z, t, y) d u d z .
$$

We define $\tilde{p}=\sum_{n=0}^{\infty} p_{n}$. Under appropriate integrability conditions, $-\tilde{p}$ is the left inverse of $\partial_{t}+\Delta_{y}+q$ [5]. We call $\tilde{p}$ the Schrödinger perturbation of $p$ by $q$, because $\partial_{t}+\Delta_{y}+q$ is an additive perturbation of $\partial_{t}+\Delta_{y}$ by the operator of multiplication by $q$. We see that $\tilde{p}(\cdot, \cdot, t, y)$ is a power series of iterates of an integral kernel operator applied to $p(\cdot, \cdot, t, y)$, which may be considered as a control function.

Estimates of such series for rather general kernels are the main subject of the paper, motivated by the results of $[5,15]$ on transition densities. The main feature of our approach is majorization of the series by means of a control function, e.g. $f$ in our main result, Theorem 3.2. The assumptions on the kernel involve local smallness (Eq. 3.1) and global boundedness (Eq. 3.2) with respect to an increasing family of absorbing sets, which add a strong transience-type property of the kernel to the picture. A representative application of Theorem 3.2 is given in Example 4.1 for the potential kernel of two 1/2-stable subordinators.

In general we neither assume Chapman-Kolmogorov conditions on the kernel nor any connection between the kernel and the control function. However, for Schrödinger perturbations, these two are related by a multiplication operation, and the setting of space-time is of special interest because it includes transition kernels. The setting is dealt with in Theorem 4.6, which is complemented by Example 4.5 and Corollary 4.11, and illustrated by Example 4.13.

Our results are analogues, and a strengthening, of Khasminski's lemma [1, 9], under a transience-type properties of the kernel. They may be regarded as extensions of Gronwall's lemma to the context of kernel operators. The results also apply to Schrödinger perturbations of continuous-time transition densities by measures. They may be used in discrete time, in fact in quite general settings, including partially ordered state spaces. In a related paper [7] we use different methods to obtain slightly more specific estimates for Schrödinger perturbations of kernels on space-time by functions.

The paper is composed as follows. In Section 2 we consider integral kernels on absorbing sets. In Section 3 we prove estimates of von Neumann series for such kernels in presence of a control function. In Section 4 we give the application to Schrödinger perturbations of the potential kernel of two subordinators. We also discuss the local smallness and global boundedness for continuous-time kernels, with focus on transition kernels and singular perturbations, including perturbations by measures.

\section{Kernels and Absorbing Sets}

Let $(E, \mathcal{E})$ be a measurable space and let $K$ be a kernel on $(E, \mathcal{E})$ [10]. That is, $K$ : $E \times \mathcal{E} \rightarrow[0, \infty]$, each $K(x, \cdot)$ is a measure on $(E, \mathcal{E})$, and each function $K(\cdot, B)$ is 
$\mathcal{E}$-measurable. We write $f \in \mathcal{E}^{+}$if $f: E \rightarrow[0, \infty]$ and $f$ is $\mathcal{E}$-measurable. For $f \in \mathcal{E}^{+}$ we let

$$
K f(x)=\int f(y) K(x, d y), \quad x \in E,
$$

and call this $K$ a kernel operator. The operator is additive, positively homogeneous, and $K f_{n}(x) \uparrow K f(x)$ whenever $f_{n} \uparrow f$. Conversely, every map from $\mathcal{E}^{+}$to $\mathcal{E}^{+}$having these properties is of the form 2.1, see [10]. For instance, if $q \in \mathcal{E}^{+}$, then the multiplication by $q$,

$$
q f(x):=q(x) f(x), \quad x \in E, f \in \mathcal{E}^{+},
$$

is a kernel operator. This is a simple but ambiguous notation, and it should always be clear from the context which meaning of $q$ we have in mind (the function or the multiplication operator). The composition of kernel operators $K$ and $L$ on $\mathcal{E}^{+}$ and the composition of kernels, $K L(x, B)=\int L(y, B) K(x, d y)$ on $(E, \mathcal{E})$, agree in the sense of Eq. 2.1, and so the composition of kernels is associative. We will often consider the multiplication by $1_{A}$, the indicator function of $A \in \mathcal{E}$.

A set $A \in \mathcal{E}$ is called $K$-absorbing, if $K\left(x, A^{c}\right)=0$ for every $x \in A$, that is if $1_{A} K 1_{A^{c}}=0$. Since $1_{E}=1_{A}+1_{A^{c}}$ and $1_{A} 1_{A^{c}}=0, A$ is $K$-absorbing if and only if

$$
1_{A} K=1_{A} K 1_{A}
$$

as kernels. Clearly, $\varnothing$ and $E$ are $K$-absorbing, and the union and intersection of countably many $K$-absorbing sets are $K$-absorbing. If $A$ is $K$-absorbing, then $A$ is $L$-absorbing for any kernel $L \leq K$.

Example 2.1 We will generalize the discussion of the Gaussian kernel from Introduction. Let $(X, \mathcal{M})$ be a measurable space. Let $E=\mathbb{R} \times X$, with the $\sigma$-algebra $\mathcal{E}$ generated by the sets $(a, b) \times A$, where $a, b \in \mathbb{R}, a<b$ and $A \in \mathcal{M}$. Let $p: E \times$ $E \rightarrow[0, \infty]$ be $\mathcal{E} \otimes \mathcal{E}$-measurable and satisfy

$$
p(s, x, t, y)=0, \quad \text { whenever } \quad s \geq t .
$$

Given a measure $\mu$ on $(E, \mathcal{E})$, we define the kernel $K^{\mu}$,

$$
K^{\mu} f(s, x):=\int p(s, x, u, z) f(u, z) d \mu(u, z), \quad(s, x) \in E, \quad f \in \mathcal{E}^{+} .
$$

We note that, for every $t \in \mathbb{R}$, the "open half-space" $(t, \infty) \times X$ and the "closed halfspace" $[t, \infty) \times X$ are absorbing for $K^{\mu}$. Thus, the first coordinate has a distinguished role for space-time $E=\mathbb{R} \times X$, which is the main setting of [7].

In many examples of interest $p$ also satisfies the Chapman-Kolmogorov equations, i.e., there is a measure $m$ on $(X, \mathcal{M})$ such that for all $s<u<t$ and $x, y \in X$,

$$
p(s, x, t, y)=\int p(s, x, u, z) p(u, z, t, y) d m(z)
$$

For the Brownian transition density, $m$ is the Lebesgue measure on $\mathbb{R}^{d}$. 
Example 2.2 Let $(T, \mathcal{T}, \rho)$ be a measure space. Let $\left\{K_{t}, t \in T\right\}$ be a family of kernels on $(E, \mathcal{E})$ such that $(t, x) \mapsto K_{t}(x, B)$ is $\mathcal{T} \otimes \mathcal{E}$-measurable for each $B \in \mathcal{E}$. Then $K:=\int K_{t} \rho(d t)$ is a kernel. Furthermore, if $A \in \mathcal{E}$ is $K_{t}$-absorbing for every $t \in T$, then $A$ is also $K$-absorbing.

For instance, let $\alpha \in(0,2)$ and let $p_{t}(y)$ be the density function of the $\alpha / 2$ stable subordinator $\left(\eta_{t}, t>0\right)$ on $\mathbb{R}$. Recall that $\left(\eta_{t}\right)$ is time-homogeneous and has independent increments, and $p_{t}(y)=0$ if $y \leq 0$. Thus the right half-lines are absorbing for the semigroup $K_{t}(x, d y):=p_{t}(y-x) d y$. We have (see, e.g., [2, V.3.4] or $[6,(1.38)])$,

$$
\int_{0}^{\infty} p_{t}(y) d t=\Gamma(\alpha / 2)^{-1} y^{\alpha / 2-1}, \quad y>0
$$

Accordingly, the right half-lines are absorbing for the potential kernel of $\left(\eta_{t}\right)$,

$$
K(x, A)=\Gamma(\alpha / 2)^{-1} \int_{A}(y-x)_{+}^{\alpha / 2-1} d y,
$$

and also for

$$
K^{\mu}(x, A)=\Gamma(\alpha / 2)^{-1} \int_{A}(y-x)_{+}^{\alpha / 2-1} \mu(d y),
$$

where $\mu$ is any Borel measure on $\mathbb{R}$.

Example 2.3 If $E$ is partially ordered and each measure $K(x, d y)$ is concentrated on $\Gamma_{x}:=\{y: x \prec y\}$, then the sets $\Gamma_{x}$ are $K$-absorbing. This is the case, e.g., for the semigroup and the potential operator of a vector of subordinators (see also Example 4.1).

Example 2.4 Let $(\mathcal{X}, \mathcal{W})$ be a balayage space [2, II.4]. Here $\mathcal{X}$ is a locally compact space with countable base, and $\mathcal{W}$ denotes the class of nonnegative hyperharmonic functions on $\mathcal{X}$ [2, III.1]. In particular, each $w \in \mathcal{W}$ is lower semicontinuous. Let $r$ be a continuous real potential on $\mathcal{X}[2$, II.5] and let $K$ be the potential kernel associated with $r$ in the sense of [2, II.6.17]. Thus, $K 1=r$, and for every bounded Borel measurable function $f \geq 0$ on $\mathcal{X}$, the function $K f$ is a continuous potential, which is harmonic outside the support of $f$, see [2, III.6.12]. Let $w \in \mathcal{W}$ and $A=$ $\{x \in \mathcal{X}: w=0\}$. Then $A$ is closed and $K$-absorbing. Indeed, let $B$ be a compact in $A^{c}$. There exists a number $c>0$ such that $c w>r$ on $B$. By the minimum principle [2, III.6.6], $c w \geq K 1_{B}$ everywhere, hence $K 1_{B}=0$ on $A$. In [2, V.1] such sets $A$ are called absorbing, too, and they have a number of equivalent characterizations, of which we mention two: (a) $A$ is closed and $P_{t}(x, \mathcal{X} \backslash A)=0$, for every $t>0$, $x \in A$, and sub-Markov semigroup $\left(P_{t}\right)_{t>0}$ having $\mathcal{W}$ as excessive functions, and (b) $A$ is closed and $P^{x}\left[X_{t} \in A \cup\{\partial\}\right]=1$ for every $t>0, x \in A$, and Markov process $\left(X_{t}, P^{x}\right)_{t>0, x \in \mathcal{X}}$ having $\mathcal{W}$ as excessive functions and $\partial$ as the cementary state. The details are given in [2, V.1.2].

Furthermore, if $A$ is any Borel set containing the (fine) superharmonic support of $r$, then $K 1_{A}=K$ [2, II.6.3], and hence $A$ is $K$-absorbing.

We will collect a few simple facts about $K$-absorbing sets. 
Lemma 2.5 Let $A$ be $K$-absorbing and $m \in \mathbb{N}$. Then

$$
1_{A} K^{m}=\left(1_{A} K\right)^{m}=1_{A} K^{m} 1_{A} .
$$

In particular, $A$ is $K^{m}$-absorbing. If furthermore $f \in \mathcal{E}^{+}$and $c \geq 0$ are such that $K f \leq$ cf on $A$, then $K^{m} f \leq c^{m} f$ on $A$.

Proof The case of $m=1$ follows from Eq. 2.2. If Eq. 2.6 holds for some $m \in \mathbb{N}$, then

$$
1_{A} K^{m+1}=1_{A} K^{m} K=\left(1_{A} K\right)^{m} 1_{A} K=\left(1_{A} K\right)^{m} 1_{A} K 1_{A}
$$

showing that Eq. 2.6 holds for $m+1$, and we can use induction. Further, $1_{A} K f \leq c f$ yields that $1_{A} K^{m} f=\left(1_{A} K\right)^{m} f \leq c^{m} f$.

Lemma 2.6 Let $A$ and $B$ be $K$-absorbing, $A \subset B$, and $m \in \mathbb{N}$. Then

$$
1_{B} K^{m} 1_{B \backslash A}=1_{B}\left(K 1_{B \backslash A}\right)^{m}=1_{B \backslash A}\left(K 1_{B \backslash A}\right)^{m} .
$$

Proof Since $A$ is $K$-absorbing, $1_{B} K 1_{B \backslash A}=1_{A} K 1_{B \backslash A}+1_{B \backslash A} K 1_{B \backslash A}=1_{B \backslash A} K 1_{B \backslash A}$. By this and Lemma 2.5 (with $B$ in place of $A$ ),

$$
\begin{aligned}
1_{B} K^{m} 1_{B \backslash A} & =\left(1_{B} K\right)^{m} 1_{B \backslash A}=\left(1_{B} K\right)^{m-1} 1_{B \backslash A} K 1_{B \backslash A}=\ldots=1_{B}\left(K 1_{B \backslash A}\right)^{m} \\
& =1_{B} K 1_{B \backslash A}\left(K 1_{B \backslash A}\right)^{m-1}=1_{B \backslash A} K 1_{B \backslash A}\left(K 1_{B \backslash A}\right)^{m-1}=1_{B \backslash A}\left(K 1_{B \backslash A}\right)^{m} .
\end{aligned}
$$

The next result is a slight modification of [12, Proposition 7.4].

Proposition 2.7 Let $A$ be K-absorbing, and let $f \in \mathcal{E}^{+}$and $c \geq 1$ be such that $\sum_{m=0}^{\infty} K^{m} f \leq c f$ on $A$. Then, for $n=0,1, \ldots$, we have

$$
K^{n} f \leq c(1-1 / c)^{n} f \quad \text { on } A .
$$

Proof Let $g=\sum_{m=0}^{\infty} K^{m} f$. We see that $g=f+K g \geq(1 / c) g+K g$ on $A$, hence $K g \leq(1-1 / c) g$ on $A$. By Lemma 2.5, for every $n \in \mathbb{N}$,

$$
K^{n} f \leq K^{n} g \leq(1-1 / c)^{n} g \leq c(1-1 / c)^{n} f \quad \text { on } A .
$$

The case of $n=0$ is trivial.

Remark 2.8 We note that, conversely, Eq. 2.8 yields that

$$
\sum_{n=0}^{\infty} K^{n} f \leq \sum_{n=0}^{\infty} c(1-1 / c)^{n} f=c^{2} f \quad \text { on } A .
$$

Thus, comparability of $\sum K^{n} f$ and $f$ is equivalent to exponential decay of $K_{n} f$.

Remark 2.9 We will consider $f=1$, the constant function. For every $a \geq 1$, there exist kernels $K$ such that $\sup _{x \in E} K 1(x)=a$, but $\sum_{m=0}^{\infty} K^{m} 1$ is bounded (see [14, 
Proposition 10.1]). Then the estimate for $K^{n} 1$ given in Eq. 2.8 is asymptotically better than the more evident upper bound by $a^{n}$.

\section{Localization on Differences of Absorbing Sets}

We first prove a discrete variant of Gronwall's lemma.

Lemma 3.1 Let $\alpha, \delta \in[0, \infty)$ and $\gamma_{1}, \ldots, \gamma_{k} \in \mathbb{R}$ be such that, for $j=1, \ldots, k$, we have $\gamma_{j} \leq \alpha+\delta \sum_{1 \leq i<j} \gamma_{i}$. Then $\gamma_{j} \leq \alpha(1+\delta)^{j-1}$ for every $j=1, \ldots, k$.

Proof We proceed by induction: $\gamma_{k+1} \leq \alpha+\delta \sum_{i=1}^{k} \alpha(1+\delta)^{i-1}=\alpha(1+\delta)^{k}$.

We fix $K$-absorbing sets $A_{1}, \ldots, A_{k}$ such that

$$
A_{1} \subset A_{2} \subset \cdots \subset A_{k} .
$$

Taking $A_{0}:=\emptyset$, for $1 \leq j \leq k$ we define slices $S_{j}:=A_{j} \backslash A_{j-1}$ and operators

$$
K_{j}:=K 1_{S_{j}}
$$

Thus, in Example 2.1 we may choose $-\infty<t_{k}<\cdots<t_{1}<\infty$, and let $A_{j}$ be the open half-space $\left(t_{j}, \infty\right) \times X$ or the closed half-space $\left[t_{j}, \infty\right) \times X$. Then each slice $S_{j}$ equals $I_{j} \times X$, where $I_{j}$ is an interval, see also Example 4.1 and Fig. 1 .

Theorem 3.2 Let $0 \leq \eta<1, \beta \geq 0$, and $f \in \mathcal{E}^{+}$be such that

$$
K_{j} f \leq \eta f \quad \text { on } S_{j}, \quad j=1, \ldots, k,
$$

and

$$
K_{j} f \leq \beta f \quad \text { on } A_{k}, \quad j=1, \ldots, k
$$

Then, for $j=1, \ldots, k$,

$$
\sum_{m=0}^{\infty} K^{m} f \leq \frac{1}{1-\eta}\left(1+\frac{\beta}{1-\eta}\right)^{j-1} f \quad \text { on } S_{j} .
$$

Proof Let $n \in \mathbb{N}$ and $g_{n}:=\sum_{m=0}^{n} K^{m} f$. For $j=1, \ldots, k$, we (recursively) define

$$
\gamma_{j}:=\frac{1}{1-\eta}\left(1+\beta \sum_{1 \leq i<j} \gamma_{i}\right)
$$

We will prove by induction that $g_{n} \leq \gamma_{j} f$ on $S_{j}$. Let $1 \leq j \leq k$, and

$$
g_{n} \leq \gamma_{i} f \quad \text { on } S_{i}, \quad \text { for every } 1 \leq i<j .
$$

Trivially, this assumption is satisfied for $j=1$. By Eq. 3.2, $K f \leq k \beta f$ on $A_{k}$. By Lemma 2.5 we obtain a rough bound, $g_{n} \leq \sum_{m=0}^{n}(k \beta)^{m} f$ on $A_{k}$. Let $\gamma \geq 0$ be the 
smallest real number such that $g_{n} \leq \gamma f$ on $S_{j}$. If $j<l \leq k$, then $1_{S_{j}} K_{l}=0$. By Eqs. 3.5 and 3.2 for all $x \in S_{j}$ we have,

$$
\begin{aligned}
g_{n}(x) & \leq f(x)+K g_{n}(x)=f(x)+\sum_{i=1}^{j} K_{i} g_{n}(x) \\
& \leq f(x)+\sum_{i=1}^{j-1} \gamma_{i} K_{i} f(x)+\gamma K_{j} f(x) \leq\left(1+\beta \sum_{i=1}^{j-1} \gamma_{i}\right) f(x)+\gamma \eta f(x) .
\end{aligned}
$$

Thus $\gamma \leq \gamma_{j}$ (see Eq. 3.4), $g_{n} \leq \gamma_{j} f$ on $S_{j}$, and the result follows by Lemma 3.1.

Remark 3.3 We shall refer to Eq. 3.1 as local smallness and to Eq. 3.2 as global boundedness. In many important cases, the local smallness already implies the global boundedness with $\beta=\eta$. In particular, it is so in Example 2.4, if $f, 1 \in \mathcal{W}$. This follows from the minimum principle [2, III.6.6] applied to the functions $\eta f-K 1_{L} \min \{f, n\}$, for compacts sets $L \subset S_{j}$ and $n \in \mathbb{N}$. It is also true in Example 2.1 provided $f=p(\cdot, \cdot, t, y)$, each $A_{j}$ is a half-space, $\mu$ does not charge the "hyperplanes" $\{t\} \times X, t \in \mathbb{R}$, and the Chapman-Kolmogorov equations are satisfied, see Lemma 4.9 below.

The following result is motivated by Proposition 2.7 and Remark 2.8.

Corollary 3.4 Assume $c>1$ and $N \in \mathbb{N}$ are such that $\eta:=c(1-1 / c)^{N}<1$. Let $\beta \geq 0$ and $f \in \mathcal{E}^{+}$be such that $K f \leq \beta f$ on $A_{k}$ and

$$
\sum_{m=0}^{\infty} K_{j}^{m} f \leq c f \quad \text { on } S_{j}
$$

for every $1 \leq j \leq k$. Then, for every $1 \leq j \leq k$,

$$
\sum_{m=0}^{\infty} K^{m} f \leq\left(\sum_{n=0}^{N-1} \beta^{n}\right) \frac{1}{1-\eta}\left(1+\frac{\beta}{1-\eta}\right)^{j-1} f \quad \text { on } S_{j} .
$$

If Eq. 3.6 holds on $A_{k}$ for every $1 \leq j \leq k$, then $K f \leq c k f$ on $A_{k}$.

Proof Let $1 \leq j \leq k$. Since each $K_{j}^{m} f$ vanishes on $A_{j-1}$, Eq. 3.6 means that $\sum_{m=0}^{\infty} K_{j}^{m} f \leq c f$ on $A_{j}$. By a remark following Eq. 2.2, $A_{j}$ is $K_{j}$-absorbing. By Lemma 2.6 and Proposition 2.7,

$$
\left(K^{N}\right)\left(1_{S_{j}} f\right)=\left(K_{j}\right)^{N} f \leq \eta f \quad \text { on } A_{j} .
$$

An application of Theorem 3.3 yields that

$$
\sum_{m=0}^{\infty}\left(K^{N}\right)^{m} f \leq \frac{1}{1-\eta}\left(1+\frac{\beta}{1-\eta}\right)^{j-1} f \quad \text { on } S_{j} .
$$

By Lemma $2.5, \sum_{n=0}^{N-1} K^{n} f \leq \sum_{n=0}^{N-1} \beta^{n} f$ on $A_{k}$. We finally note that

$$
\sum_{m=0}^{\infty} K^{m} f=\sum_{m=0}^{\infty}\left(K^{N}\right)^{m}\left(\sum_{n=0}^{N-1} K^{n} f\right) \leq\left(\sum_{n=0}^{N-1} \beta^{n}\right) \sum_{m=0}^{\infty}\left(K^{N}\right)^{m} f .
$$


If Eq. 3.6 holds even on $A_{k}$ for $1 \leq j \leq k$, then $K f \leq \sum_{j=1}^{k} K_{j} f \leq c k f$ on $A_{k}$, and we can take $\beta=c k$.

\section{Examples and Applications}

We may use Theorem 3.2 to estimate Schrödinger-type perturbations of kernels. As a rule, auxiliary estimates of the kernels are needed for such applications.

Example 4.1 For $t>0$ and $x \in \mathbb{R}$ we define

$$
f_{t}(x)=\left\{\begin{array}{cc}
(4 \pi)^{-1 / 2} t x^{-3 / 2} e^{-t^{2} /(4 x)}, & \text { if } x>0 \\
0, & \text { else }
\end{array}\right.
$$

the density function of the 1/2-stable subordinator. By [20, Example 2.13],

$$
\int_{0}^{\infty} f_{t}(x) e^{-u x} d x=e^{-t u^{1 / 2}}, \quad u \geq 0 .
$$

For $\phi \in C_{c}^{\infty}(\mathbb{R})$ (smooth compactly supported real-valued functions on $\mathbb{R}$ ) we let

$$
P_{t} \phi(x)=\int_{0}^{\infty} \phi(x+z) f_{t}(z) d z, \quad x \in \mathbb{R} .
$$

The generator of the semigroup $\left(P_{t}\right)$ is the Weyl fractional derivative,

$$
\begin{aligned}
\partial^{1 / 2} \phi(x) & =\int_{0}^{\infty}(4 \pi)^{-1 / 2} z^{-3 / 2}(\phi(x+z)-\phi(x)) d z \\
& =\pi^{-1 / 2} \int_{0}^{\infty} z^{-1 / 2} \phi^{\prime}(x+z) d z .
\end{aligned}
$$

Schrödinger perturbations of $\partial^{\beta}$ for $\beta \in(0,1)$ were considered in [7]. We shall discuss those for the generator $L=\partial_{s}^{1 / 2}+\partial_{x}^{1 / 2}$ of the semigroup of two independent $1 / 2$ stable subordinators,

$$
T_{t} \varphi(s, x)=\int_{0}^{\infty} \int_{0}^{\infty} \varphi(s+u, x+z) f_{t}(u) f_{t}(z) d u d z, \quad s, x \in \mathbb{R} .
$$

Here and below, $\varphi \in C_{c}^{\infty}(\mathbb{R} \times \mathbb{R})$. For $s, x \in \mathbb{R}$ we have

$$
\varphi(s, x)=-\int_{0}^{\infty} \frac{d}{d t} T_{t} \varphi(s, x) d t=-\int_{0}^{\infty} T_{t} L \varphi(s, x) d t .
$$

In view of Eq. 4.1 we need to calculate the potential kernel $\int_{0}^{\infty} T_{t} d t$. Let

$$
\begin{aligned}
\kappa(s, x) & =\int_{0}^{\infty} f_{t}(s) f_{t}(x) d t \\
& =\left\{\begin{array}{cc}
(4 \pi)^{-1 / 2}(s+x)^{-3 / 2}, & \text { if } s, x>0, \\
0, & \text { else, }
\end{array}\right.
\end{aligned}
$$

where the latter formula follows from direct integration. Define

$$
\kappa(s, x, u, z)=\kappa(u-s, z-x), \quad s, x, u, z \in \mathbb{R} .
$$


By Eq. 4.1, we obtain

$$
\int_{\mathbb{R}} \int_{\mathbb{R}} \kappa(s, x, u, z)\left(\partial_{u}^{1 / 2}+\partial_{z}^{1 / 2}\right) \varphi(u, z) d u d z=-\varphi(s, x), \quad s, x \in \mathbb{R} .
$$

We observe a 3G-type inequality: if $s<u<t$ and $x<z<y$, then

$$
\kappa(s, x, t, y) \leq \kappa(s, x, u, z) \wedge \kappa(u, z, t, y) \leq 2 \sqrt{2} \kappa(s, x, t, y),
$$

since $t-s+y-x \geq(u-s+z-x) \vee(t-u+y-z) \geq(t-s+y-x) / 2$. Thus,

$$
\kappa(s, x, u, z) \kappa(u, z, t, y) \leq 2 \sqrt{2} \kappa(s, x, t, y)[\kappa(s, x, u, z) \vee \kappa(u, z, t, y)],
$$

where $s<u<t, x<z<y$, and this is sharp, since Eq. 4.3 also yields

$$
\kappa(s, x, u, z) \kappa(u, z, t, y) \geq \kappa(s, x, t, y)[\kappa(s, x, u, z)+\kappa(u, z, t, y)] / 2 .
$$

For $0<p<1 / 2$ and a number $c>0$ we let

$$
q_{0}(u, z)=\left\{\begin{array}{cc}
c(u+z)^{-p}, & \text { if } u, z>0, \\
0, & \text { else. }
\end{array}\right.
$$

We consider $0 \leq q \leq q_{0}$ and the kernel

$$
K f(s, x):=\int_{\mathbb{R}^{2}} \kappa(s, x, u, z) q(u, z) f(u, z) d z d u .
$$

We will use Theorem 3.2 to compare $\kappa$ with $\tilde{\kappa}$ defined as

$$
\tilde{\kappa}=\sum_{m=0}^{\infty}(\kappa q)^{m} \kappa,
$$

or, more precisely,

$$
\tilde{\kappa}(s, x, t, y)=\sum_{m=0}^{\infty} K^{m} f(s, x),
$$

where we fix $t, y \in \mathbb{R}$ and denote (the control function)

$$
f(s, x):=\kappa(s, x, t, y) .
$$

We let $s<t$ and $x<y$, because otherwise $\tilde{\kappa}(s, x, t, y)=0=\kappa(s, x, t, y)$. Furthermore, we assume that $t+y>0$, else $\tilde{\kappa}(s, x, t, y)=\kappa(s, x, t, y)$. Let $h>0$ and $k \geq 1$ be such that $(k-1) h \leq t+y<k h$ ( $h$ is defined later on). For $j=0, \ldots, k$, we let $a_{j}=(k-j) h$. For $j=1, \ldots, k-1$, we define $A_{j}=\left\{(u, z): u+z \geq a_{j}\right\}$. We also let $A_{0}=\emptyset$, and $A_{k}=\mathbb{R}^{2}$. The sets $A_{j}$ are increasing and absorbing. For $j=1, \ldots, k$, we define $S_{j}=A_{j} \backslash A_{j-1}$, see Fig. 1 . We will call $\{(u, z): u+z=\xi\}, \xi \in \mathbb{R}$, the level lines. We define $K_{j}=K 1_{S_{j}}$, as in Theorem 3.2. We have

$$
\begin{aligned}
& K_{j} f(s, x) / f(s, x) \\
& \quad \leq 2 \sqrt{2} c \int_{S_{j} \cap\{s \leq u \leq t, x \leq z \leq y\}}\left[(u+z-s-x)^{-3 / 2}+(t+y-u-z)^{-3 / 2}\right](u+z)^{-p} d z d u .
\end{aligned}
$$




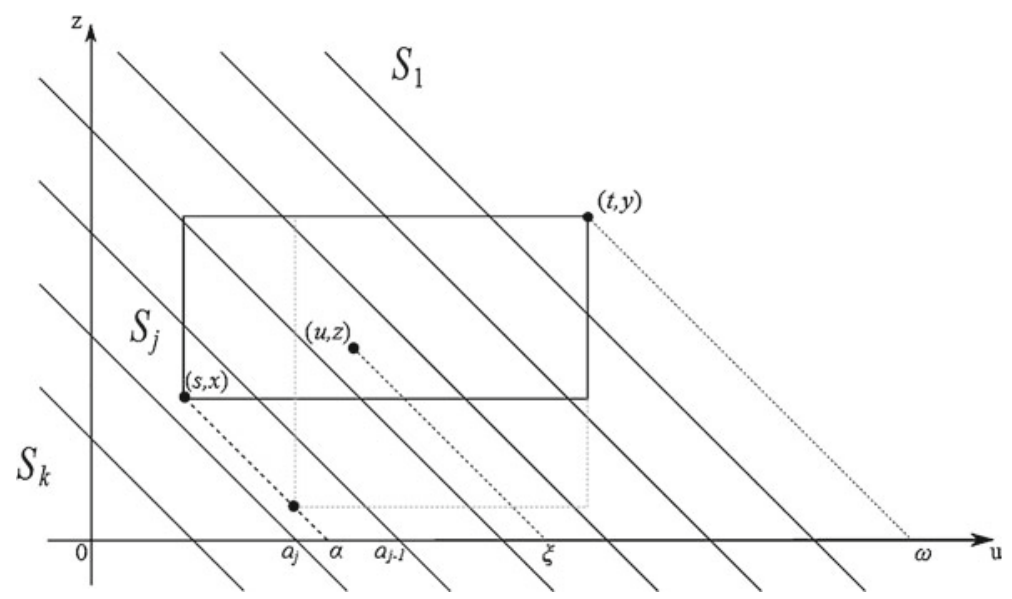

Fig. 1 Notation for Example 4.1

We will estimate the right-hand side of Eq. 4.6. Denote $\alpha=s+x, \omega=t+y$ and $\xi=u+z$. Let $\alpha<a_{j-1}$ and $\omega>a_{j}$ (otherwise the integral is zero). The integrand is constant along the level lines. The integral is the largest when $\left\{(s, u) \in \mathbb{R}^{2}: s \leq u \leq\right.$ $t, x \leq z \leq y\}$ is a square, because the square's intersections with the level lines have the largest length, namely $\sqrt{2}[(\xi-\alpha) \wedge(\omega-\xi)]$, see Fig. 1. Taking this into account or substituting $\xi=u+z, \eta=(u-z) / 2$, we bound the integral in Eq. 4.6 by

$$
\begin{aligned}
& \int_{\alpha \vee a_{j}}^{\omega \wedge a_{j-1}}(\xi-\alpha)(\xi-\alpha)^{-3 / 2} \xi^{-p}+(\omega-\xi)(\omega-\xi)^{-3 / 2} \xi^{-p} d \xi \\
& \quad \leq \int_{a_{j}}^{a_{j-1}}\left(\xi-a_{j}\right)^{-1 / 2-p}+\left(a_{j-1}-\xi\right)^{-1 / 2}\left(\xi-a_{j}\right)^{-p} d \xi \\
& \quad=[B(1 / 2-p, 1)+B(1 / 2,1-p)]\left(a_{j-1}-a_{j}\right)^{1 / 2-p},
\end{aligned}
$$

where $B$ is the Euler beta function.

By Theorem 3.2, if we let $\eta=\beta=2 \sqrt{2} c[B(1 / 2-p, 1)+B(1 / 2,1-p)] h^{1 / 2-p}<1$ (the inequality determines $h$ ), then

$$
\tilde{\kappa}(s, x, t, y) \leq\left(\frac{1}{1-\eta}\right)^{j} \kappa(s, x, t, y) \quad \text { for } \quad(s, x) \in S_{j} .
$$

In fact, $j<k+1-(s+x) / h \leq(t+y-s-x) / h+2$. We see that $\kappa$ and $\tilde{\kappa}$ are locally comparable. We also note that the first coordinate does not play a distinguished role here, in contrast to the examples in [7] and below. Finally, $\tilde{\kappa}$ may be considered a Schrödinger perturbation of $\kappa$, because

$$
\int_{\mathbb{R} \times \mathbb{R}} \tilde{\kappa}(s, x, u, z)\left[\partial_{z}^{1 / 2}+\partial_{u}^{1 / 2}+q(u, z)\right] \varphi(u, z) d z d u=-\varphi(s, x),
$$

for $s, x \in \mathbb{R}$ and $\phi \in C_{c}^{\infty}(\mathbb{R} \times \mathbb{R})$. The identity 4.8 is proved by using [7, (31)]. Indeed, the absolute integrability of the integrals in [7, (31)] follows by considering the 
supports of the involved functions (we leave details to the reader). We also wish to note that if $q_{0}(u, z)$ depends only on $u$ or $u \wedge z$, then it is more convenient to consider absorbing sets $\left\{(u, z) \in \mathbb{R}^{2}: u>s\right\}$ or $\left\{(u, z) \in \mathbb{R}^{2}: u>s, z>x\right\}$, correspondingly.

In the remainder of the paper we shall adopt the setting of Example 2.1. More precisely, we consider the space-time $E=\mathbb{R} \times X$, with the product $\sigma$-algebra $\mathcal{E}$, and an $\mathcal{E} \times \mathcal{E}$-measurable function $p \geq 0$ on $E \times E$ such that Eq. 2.3 holds, but we do $n o t$ assume Eq. 2.5. For a measure $\mu$ on $(E, \mathcal{E})$ we define kernel $K^{\mu}$ by Eq. 2.4. Motivated by the discussion in Section 1 and Example 2.1, we let

$$
p^{\mu}=\sum_{n=0}^{\infty} p_{n}^{\mu}
$$

where $p_{0}^{\mu}=p$, and the positive functions $p_{1}^{\mu}, p_{2}^{\mu} \ldots$ on $E \times E$ are defined as follows,

$$
p_{n}^{\mu}(s, x, t, y):=\int p_{n-1}^{\mu}(s, x, u, z) p(u, z, t, y) d \mu(u, z) .
$$

By induction, $p_{n}^{\mu}(s, x, t, y)=0$ for $n \geq 0,(s, x),(t, y) \in E$, if $s \geq t$. According to Introduction, we perturb $p$ by the measure $\mu$ (but see Example 4.5, too). We regard $(t, y)$ as fixed when iteratively transforming $f(s, x):=p(s, x, t, y)$ by $K^{\mu}$ :

$$
p_{n}^{\mu}(\cdot, \cdot, t, y)=\left(K^{\mu}\right)^{n} p(\cdot, \cdot, t, y) .
$$

Remark 4.2 Similar perturbations may be studied for signed measures, say $v$. We clearly have $\left|p^{v}\right| \leq p^{\mu}$, where $\mu=v_{-}+v_{+}$is the variation measure of $v$. We will not further concern ourselves with signed kernels or functions in this paper.

In Examples 4.3, 4.4 and 4.5 we will additionally suppose that $p$ is a transition density, that is, the Chapman-Kolmogorov Eq. 2.5 hold with respect to a $\sigma$-finite measure $m$ on $X$.

Example 4.3 Let $\rho \geq 0$ be a Radon measure on $\mathbb{R}$ having no atoms, and let $\mu:=\rho \otimes m$. Then, for all $(s, x),(t, y) \in E$ and $n \in \mathbb{N}, p_{n}^{\mu}(s, x, t, y)=\rho((s, t))^{n} p(s, x, t, y) / n !$ by induction, and we obtain the transition density

$$
p^{\mu}(s, x, t, y)=e^{\rho((s, t))} p(s, x, t, y) .
$$

Example 4.4 Let $\eta>0, u_{0} \in \mathbb{R}, \mu:=\eta \varepsilon_{u_{0}} \otimes m$. Here $\varepsilon_{u_{0}}(f)=f\left(u_{0}\right)$ is the Dirac measure. Then $\mu$ is concentrated on the "hyperplane" $\left\{u_{0}\right\} \times E$, and for $(s, x)$, $(t, y) \in E$ we have by Eq. 2.5,

$$
p_{1}^{\mu}(s, x, t, y)=\int p(s, x, u, z) p(u, z, t, y) d \mu(u, z)= \begin{cases}\eta p(s, x, t, y), & \text { if } s<u_{0}<t, \\ 0, & \text { otherwise }\end{cases}
$$

For $n=2,3, \ldots$ and all $(s, x),(t, y) \in E$, we obtain $p_{n}^{\mu}(s, x, t, y)=0$, hence

$$
p^{\mu}(s, x, t, y):=\sum_{n=0}^{\infty} p_{n}^{\mu}(s, x, t, y)= \begin{cases}(1+\eta) p(s, x, t, y), & \text { if } s<u_{0}<t \\ p(s, x, t, y), & \text { otherwise }\end{cases}
$$

There is, however, an alternative approach to perturbations by such measures. 
Example 4.5 Let $u_{0} \in \mathbb{R}$ and $\mu:=\varepsilon_{u_{0}} \otimes m$. For $g \in \mathcal{E}^{+}$we define

$$
K g(s, x)= \begin{cases}0, & \text { if } s>u_{0}, \\ g(s, x), & \text { if } s=u_{0}, \\ \int_{\mathbb{R}^{d}} p\left(s, x, u_{0}, z\right) g\left(u_{0}, z\right) d m(z), & \text { if } s<u_{0} .\end{cases}
$$

Let $t>u_{0}$ and $y \in \mathbb{R}^{d}$ be fixed. We consider $f(s, x)=p(s, x, t, y),(s, x) \in E$. By Chapman-Kolmogorov equations, $K f(s, x)=1_{s \leq u_{0}} p(s, x, t, y)$. By induction, $K^{n} f(s, x)=1_{s \leq u_{0}} p(s, x, t, y)$, for $n=1,2, \ldots$ If $0<\eta<1$, then

$$
\tilde{p}(s, x, t, y):=\sum_{n=0}^{\infty}(\eta K)^{n} f(s, x)= \begin{cases}(1-\eta)^{-1} p(s, x, t, y), & \text { for } s \leq u_{0}, \\ p(s, x, t, y), & \text { otherwise }\end{cases}
$$

whereas $\eta \geq 1$ leads to explosion of $\tilde{p}$. We observe that $\tilde{p}$ satisfies ChapmanKolmogorov equations, but not $p^{\mu}$ defined in Example 4.4.

More generally, for an arbitrary Radon measure $\rho$ on $\mathbb{R}$, we let

$$
K g(s, x)=\rho(\{s\}) g(s, x)+\int_{(s, \infty)} \int_{X} p(s, x, u, z) g(u, z) d m(z) \rho(d u) .
$$

We note that $K=K^{\rho \otimes m}$ (see Eq. 2.4), if $\rho$ has no atoms. On one hand this motivates our interest in $K^{\mu}$ later in this section. On the other hand, atoms are intrinsically related to the estimates obtained in $[7,15]$ and in Theorem 4.6 below, because they produce inflation of mass very close to that given by the estimates. Indeed, let us fix numbers $u_{1}<u_{2}<\ldots<u_{k}$, and let $\rho=\varepsilon_{u_{1}}+\varepsilon_{u_{2}}+\ldots+\varepsilon_{u_{k}}$. Assume that $u_{k}<t$. We have $K f(s, x)=L(s) p(s, x, t, y)$, with $f$ as before and

$$
L(s):=\#\left\{1 \leq i \leq k: u_{i} \geq s\right\} .
$$

By induction we verify that

$$
\begin{aligned}
K^{n} f(s, x) & =\#\left\{\left(i_{1}, \ldots, i_{n}\right): s \leq u_{i_{1}} \leq \ldots \leq u_{i_{n}}\right\} p(s, x, t, y) \\
& =\left(\begin{array}{c}
L(s)+n-1 \\
n
\end{array}\right) p(s, x, t, y) .
\end{aligned}
$$

Notably, a similar combinatorics is triggered by gradient perturbation series in [17, Lemma 5]. If $0<\eta<1$, then, by Taylor series expansion [15, p. 51],

$$
\tilde{p}(s, x, t, y):=\sum_{n=0}^{\infty}(\eta K)^{n} f(s, x, t, y)=\left(\frac{1}{1-\eta}\right)^{L(s)} p(s, x, t, y) .
$$

This should be compared with Theorem 4.6 below.

We now return to functions $p$ as specified before Eq. 4.9, i.e., we do not assume Chapman-Kolmogorov conditions, unless we explicitly say otherwise.

Let $I \subset \mathbb{R}$ be an interval and let

$$
\mu_{I}(A):=\mu(A \cap(I \times X)), \quad A \in \mathcal{E} .
$$

For $n=0,1,2, \ldots$, we denote (see above in this section)

$$
p_{n}:=p_{n}^{\mu} \quad \text { and } \quad p_{n}^{I}:=p_{n}^{\mu_{I}} .
$$

We also note that $p_{n}(s, x, t, y)=p_{n}^{(s, t)}(s, x, t, y)$, which follows by induction. 
The half-spaces $(t, \infty) \times X$ and $[t, \infty) \times X$ are $K^{\mu}$-absorbing for $t \in \mathbb{R}$. The differences of such sets are of the form $I \times X$, where $I$ is an interval. For $I, J \subset \mathbb{R}$, we write $I \prec J$, if $s<t$ for all $s \in I$ and $t \in J$.

Theorem 4.6 Let $-\infty<r<t<\infty, y \in X, \eta \in[0,1)$. Suppose that $[r, t)$ is the union of intervals $I_{k} \prec \cdots \prec I_{1}$, such that for all $j=1, \ldots, k$, and $x \in X$,

$$
\int_{I_{j} \times X} p(s, x, u, z) p(u, z, t, y) d \mu(u, z) \leq \eta p(s, x, t, y), \quad r \leq s<t .
$$

Then, for $j=1, \ldots, k$ and $x \in X$,

$$
p^{\mu}(s, x, t, y):=\sum_{n=0}^{\infty} p_{n}(s, x, t, y) \leq\left(\frac{1}{1-\eta}\right)^{j} p(s, x, t, y), \quad s \in I_{j} .
$$

Proof We may apply Theorem 3.2 to $f(s, x):=p(s, x, t, y), A_{j}=\left(I_{j} \cup \ldots \cup I_{1}\right) \times X$, $K_{j}:=K^{\mu_{I_{j}}}$, and $\beta:=\eta$, since Eq. 4.16 implies both Eqs. 3.1 and 3.2.

Corollary 4.7 Let $-\infty<r<t<\infty, y \in X, \beta \geq 0$ and $c \geq 1$. Suppose that

$$
p_{1}(s, x, t, y) \leq \beta p(s, x, t, y), \quad \text { for all } s>r, x \in X,
$$

and $[r, t)$ is a union of disjoint intervals $I_{1}, I_{2}, \ldots, I_{k}$ satisfying,

$$
\sum_{n=0}^{\infty} p_{n}^{I_{j}}(s, x, t, y) \leq c p(s, x, t, y), \quad \text { for } s \in I_{j}, x \in X \quad(1 \leq j \leq k) .
$$

Then there exists a constant $C$ such that $\sum_{n=0}^{\infty} p_{n}(s, x, t, y) \leq C p(s, x, t, y)$ for all $s \geq r$ and $x \in X$.

Proof We proceed as in the proof of Theorem 4.6, using Corollary 3.4. We let $C=$ $\left(\sum_{n=0}^{N-1} \beta^{n}\right)[1+\beta /(1-\eta)]^{k-1} /(1-\eta)$, where $\eta=c(1-1 / c)^{N}<1$.

Remark 4.8 If the inequality in Eq. 4.19 holds on $[r, \infty) \times X$, for $1 \leq j \leq k$, then

$$
p_{1}(s, x, t, y)=\sum_{j=1}^{k} p_{1}^{I_{j}}(s, x, t, y) \leq k c p(s, x, t, y), \quad s \geq r, x \in X,
$$

and Eq. 4.18 holds with $\beta=k c$.

If $p$ satisfies Eq. 2.5, then we can localize Eq. 4.16 as follows.

Lemma 4.9 Suppose that $p$ satisfies the Chapman-Kolmogorov equations. Let $(t, y) \in E, \eta \geq 0$, and let an interval $I \subset(-\infty, t)$ satisfy, for all $(s, x) \in I \times X$,

$$
\int p(s, x, u, z) p(u, z, t, y) d \mu_{I}(u, z) \leq \eta p(s, x, t, y) .
$$

Then Eq. 4.20 holds for all $(s, x) \in E$. 
Proof If $s \in I$ or $s$ is to the right of $I$, then Eq. 4.20 clearly holds, see Eq. 2.3. If $s$ is to the left of $I, a \in I, J:=[a, \infty) \cap I$, and $x \in E$, then by Eqs. 2.5 and 4.20,

$$
\begin{aligned}
\int & p(s, x, u, z) p(u, z, t, y) d \mu_{J}(u, z) \\
& =\iint p(s, x, a, w) p(a, w, u, z) p(u, z, t, y) d m(w) d \mu_{J}(u, z) \\
& \leq \eta \int p(s, x, a, w) p(a, w, t, y) d m(w)=\eta p(s, x, t, y) .
\end{aligned}
$$

So Eq. 4.20 holds, if inf $I \in I$ (take $a=\inf I$ ). If not, it follows by monotone convergence, by letting $a \in I$ approach inf $I$.

Lemma 4.10 Suppose that p satisfies the Chapman-Kolmogorov equations. Let $\eta \geq 0$ and an interval I be such that, for all $s, t \in I$ and $x, y \in X$,

$$
\int p(s, x, u, z) p(u, z, t, y) d \mu_{I}(u, z) \leq \eta p(s, x, t, y) .
$$

Then Eq. 4.21 holds for all $(s, x),(t, y) \in E$.

Proof Let us fix $(t, y) \in E$. By Eq. 2.3 we may replace $I$ by $I \cap(-\infty, t)$. An application of Lemma 4.9 finishes the proof.

Corollary 4.11 Suppose that $p$ satisfies the Chapman-Kolmogorov equations. Let $-\infty<r<t<\infty, y \in X$ and $\eta \in[0,1)$. Let $[r, t)$ be the union of intervals $I_{k} \prec \cdots \prec I_{1}$. Assume that for $j=1, \ldots, k$ and $I:=I_{j}, E q .4 .21$ holds for all $s \in I_{j}$ and $x \in X$. Then Eq. 4.17 holds for $j=1, \ldots, k$ and $x \in X$.

Proof The result follows from Theorem 4.6 and Lemma 4.10.

Remark 4.12 To prove comparability of $p$ and $p^{\mu}$ under Eq. 2.5 in specific situations, it is enough to choose intervals $I_{j}$ such that $\mu\left(E \backslash\left(I_{1} \cup \ldots \cup I_{k}\right) \times X\right)=0$, and for all $s, t \in I, x, y \in X, j=1, \ldots, k$,

$$
\int_{I_{j}} p(s, x, u, z) p(u, z, t, y) d \mu(z) \leq \eta p(s, x, t, y) .
$$

If Eq. 4.21 fails, then $p^{\mu}$ may be much bigger than $p$, see Example 4.5.

Our last example is essentially from [5].

Example 4.13 We consider the Cauchy transition density on $\mathbb{R}^{d}$, i.e. we let

$$
p(s, x, t, y)= \begin{cases}c_{d}(t-s)\left[(t-s)^{2}+|y-x|^{2}\right]^{-(d+1) / 2}, & \text { if } s<t, \\ 0, & \text { if } s \geq t .\end{cases}
$$

We observe the following power-type asymptotics of $p$ :

$$
p(s, x, t, y) \approx \frac{t-s}{|y-x|^{d+1}} \wedge(t-s)^{-d}, \quad x, y \in \mathbb{R}^{d}, s<t,
$$


where $L \approx R$ means that $L / R$ is bounded away from zero and infinity. In consequence, there is a constant $c$ depending only on $d$, such that

$$
p(s, x, u, z) \wedge p(u, z, t, y) \leq c p(s, x, t, y), \quad x, z, y \in \mathbb{R}^{d}, s, u, t \in \mathbb{R},
$$

see the 3P Theorem in [3]. For numbers $a, b \geq 0$ we have $a b=(a \vee b)(a \wedge b)$ and $a \vee b \leq a+b$. Therefore Eq. 4.24 yields the following variant:

$$
p(s, x, u, z) p(u, z, t, y) \leq c p(s, x, t, y)[p(s, x, u, z)+p(u, z, t, y)],
$$

and we obtain

$$
p_{1}(s, x, t, y) \leq c p(s, x, t, y) \int_{\mathbb{R}^{d}} \int_{s}^{t}[p(s, x, u, z)+p(u, z, t, y)] d \mu(u, z) .
$$

Assume that $\mu$ is of Kato class, to wit,

$$
k(h):=\sup _{x, y \in \mathbb{R}^{d}, s<t \leq s+h} \int_{\mathbb{R}^{d}} \int_{s}^{t}[p(s, x, u, z)+p(u, z, t, y)] d \mu(u, z) \rightarrow 0 \quad \text { as } h \rightarrow 0 .
$$

Let $h>0$ and $\eta:=c k(h)<1$. If $s+(j-1) h<t \leq s+j h$, where $j$ is a natural number, then, by Corollary 4.11 , for all $x, y \in \mathbb{R}^{d}$,

$$
p^{\mu}(s, x, t, y) \leq\left(\frac{1}{1-\eta}\right)^{j} p(s, x, t, y) \leq\left(\frac{1}{1-\eta}\right)^{1+(t-s) / h} p(s, x, t, y) .
$$

This is a special case of [15, Theorem 1]. In particular, if $d>1$, then, by Eq. 4.23,

$$
\int_{s}^{t} p(s, x, u, z) d u \approx|z-x|^{1-d} \wedge\left[(t-s)^{2}|z-x|^{-d-1}\right], \quad x, y \in \mathbb{R}^{d}, s<t,
$$

and if $|d \mu(u, z)| \leq|z|^{-1+\varepsilon} d z d u$ for some $\varepsilon \in(0,1]$, then $\mu$ is of Kato class.

We refer the reader to [5] for a comparison of different Kato conditions. We also refer to [1] for a discussion of discontinuous multiplicative functionals of Markov processes, which bring some analogies with Example 4.5. We also wish to mention recent results [4, 8] for non-local Schrödinger-type perturbations (see [18] and [21], too). Schrödinger perturbations of the Gaussian transition density are studied in $[19,22]$, see also [11]. We refer to $[3,5,13,14,16]$ for further instances, applications and forms of the $3 \mathrm{P}$ (or $3 \mathrm{G}$ ) inequality 4.24. In a related paper [7] we present a more specialized approach to Schrödinger perturbations by functions for transition densities, transition probabilities and general integral kernels in continuous time.

Open Access This article is distributed under the terms of the Creative Commons Attribution License which permits any use, distribution, and reproduction in any medium, provided the original author(s) and the source are credited.

\section{References}

1. Beznea, L., Boboc, N.: Feynman-Kac formula for left continuous additive functionals and extended Kato class measures. Potential Anal. 30(2), 139-164 (2009)

2. Bliedtner, J., Hansen, W.: Potential Theory-An Analytic and Probabilistic Approach to Balayage. Universitext. Springer, Berlin (1986) 
3. Bogdan, K., Jakubowski, T.: Estimates of heat kernel of fractional Laplacian perturbed by gradient operators. Commun. Math. Phys. 271(1), 179-198 (2007)

4. Bogdan, K., Sydor, S.: On nonlocal perturbations of integral kernels (2012). arXiv:1205.4571

5. Bogdan, K., Hansen, W., Jakubowski, T.: Time-dependent Schrödinger perturbations of transition densities. Stud. Math. 189(3), 235-254 (2008)

6. Bogdan, K., Byczkowski, T., Kulczycki, T., Ryznar, M., Song, R., Vondraček, Z.: Potential analysis of stable processes and its extensions. In: Lecture Notes in Mathematics, vol. 1980. Springer, Berlin (2009)

7. Bogdan, K., Jakubowski, T., Sydor, S.: Estimates of perturbation series for kernels. J. Evol. Equ. 1-12 (2012)

8. Chen, Z., Kim, P., Song, R.: Stability of Dirichlet heat kernel estimates for non-local operators under Feynman-Kac perturbation (2011). arXiv:1112.3401

9. Chung, K.L., Zhao, Z.X.: From Brownian motion to Schrödinger's equation. In: Grundlehren der Mathematischen Wissenschaften [Fundamental Principles of Mathematical Sciences], vol. 312. Springer, Berlin (1995)

10. Dellacherie, C., Meyer, P.-A.: Probabilities and potential. C. In: North-Holland Mathematics Studies, vol. 151. North-Holland, Amsterdam (1988). Potential theory for discrete and continuous semigroups, Translated from the French by J. Norris

11. Gulisashvili, A.: Classes of time-dependent measures, non-homogeneous Markov processes, and Feynman-Kac propagators. Trans. Am. Math. Soc. 360(8), 4063-4098 (2008)

12. Hansen, W.: Normalized solutions of Schrödinger equations with potentially bounded measures. Potential Anal. 21(2), 99-135 (2004)

13. Hansen, W.: Uniform boundary Harnack principle and generalized triangle property. J. Funct. Anal. 226(2), 452-484 (2005)

14. Hansen, W.: Global comparison of perturbed Green functions. Math. Ann. 334(3), 643-678 (2006)

15. Jakubowski, T.: On combinatorics of Schrödinger perturbations. Potential Anal. 31(1), 45-55 (2009)

16. Jakubowski, T., Szczypkowski, K.: Time-dependent gradient perturbations of fractional Laplacian. J. Evol. Equ. 10(2), 319-339 (2010)

17. Jakubowski, T., Szczypkowski, K.: Estimates of gradient perturbation series. J. Math. Anal. Appl. 389(1), 452-460 (2012)

18. Kim, P., Lee, Y.-R.: Generalized $3 \mathrm{G}$ theorem and application to relativistic stable process on non-smooth open sets. J. Funct. Anal. 246(1), 113-143 (2007)

19. Liskevich, V., Vogt, H., Voigt, J.: Gaussian bounds for propagators perturbed by potentials. J. Funct. Anal. 238(1), 245-277 (2006)

20. Sato, K.-i.: Lévy processes and infinitely divisible distributions. In: Cambridge Studies in Advanced Mathematics, vol. 68. Cambridge University Press, Cambridge (1999). Translated from the 1990 Japanese original, Revised by the author

21. Wang, C.: On estimates of the density of Feynman-Kac semigroups of $\alpha$-stable-like processes. J. Math. Anal. Appl. 348(2), 938-970 (2008)

22. Zhang, Q.S.: A sharp comparison result concerning Schrödinger heat kernels. Bull. Lond. Math. Soc. 35(4), 461-472 (2003) 